\title{
André Berger, de la théorie astronomique au réchauffement global et au développement durable
}

\section{Propos recueillis par Jacques-Louis de Beaulieu et Claude Millier}

\author{
André Berger ${ }^{1}$, Jacques-Louis de Beaulieu ${ }^{2}$, Claude Millier ${ }^{3}$ \\ 1 Climatologue, Université catholique de Louvain, Centre de recherche sur la Terre et le climat Georges Lemaître, \\ B-1348 Louvain-la-Neuve, Belgique \\ 2 Paléoécologue, CNRS, UMR7263 IMBE, Université Aix-Marseille (UMA), 13545 Aix-en-Provence, France \\ 3 Biomathématicien, président de l'association NSS-Dialogues, 92001 Nanterre cedex, France
}

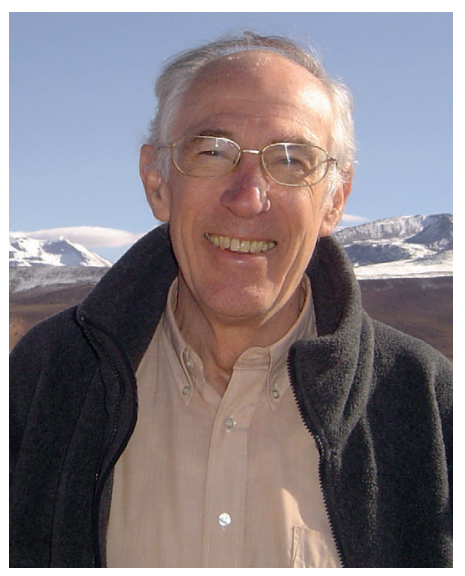

André Berger est né en 1942 à Acoz, petit village du centre de la Belgique. Après une licence ès sciences mathématiques de l'Université catholique de Louvain (UCL) en 1964, il a obtenu un master en sciences météorologiques au Massachusetts Institute of Technology (USA) en 1971, puis soutenu une thèse de doctorat en sciences de l'UCL. Aujourd'hui professeur émérite et chercheur honoraire à l'UCL, il a consacré sa vie à l'enseignement de la météorologie et de la climatologie dynamique, mais surtout à la recherche sur les climats anciens, ce qui l'a conduit à s'intéresser à toutes les facettes de cette science multidisciplinaire, y compris aux impacts du réchauffement global et de la pollution sur l'environnement et la société. Il a connu une carrière internationale et reçu de nombreuses récompenses (docteur honoris causa de l'Université d'AixMarseille III, de l'Université Paul Sabatier de Toulouse et de la Faculté polytechnique de Mons, notamment). Il est, par ailleurs, membre de plusieurs académies, dont l'Académie des sciences de Paris. Enfin, ces dernières années, il s'est investi dans la lutte contre le changement climatique en s'adressant particulièrement au grand public. Parmi ses multiples publications, signalons : Berger, A., 1992. Le Climat de la Terre: un passé pour quel avenir ?, Bruxelles, De Boeck Université ; Abel, O., Bard, É., Berger, A., Besnier, J.-M, Guesnerie, R., Serres, M. (Ed. Léna, P., Oborne, M.), 2009. Éthique et changement climatique, Paris, Le Pommier ; Berger, A., Imbrie, J., Hays, J., Kukla, G., Saltzman, B. (Eds), 1984. Milankovitch and the Climate: Understanding the Response to Orbital Forcing, Dordrecht \& Boston, D. Reidel Publishing Co.

\section{Construction d'une science}

NSS : Le réchauffement de la planète, révélé par les climatologues, est devenu depuis le sommet de Rio en 1992 une question politique majeure. Les problématiques cruciales de stratégies d'atténuation ou d'adaptation mobilisent tous les compartiments des sciences de l'environnement et de la société et constituent donc un carrefour interdisciplinaire que NSS n'a pas manqué

Auteur correspondant: J.-L. de Beaulieu, jacques-louis.debeaulieu@imbe.fr d'aborder, en particulier dans son dossier « Adaptation aux changements climatiques» (NSS, 10, 3 [2010]). Récemment, les doutes émis par les «climatosceptiques" sur la validité des bases scientifiques et des résultats des modèles prédictifs, ainsi que sur la qualité des travaux du GIEC, ont jeté le trouble dans l'opinion, même si ces doutes ont été repoussés notamment par les Académies des sciences de nombreux pays.

Pour éclairer les conditions de la construction des modèles climatiques prédictifs, NSS a souhaité s'entretenir avec vous, André Berger. En tant que mathématicien, vous 
avez, en effet, validé la théorie astronomique des climats, ce qui a fait de vous l'un des fondateurs de la climatologie moderne. Vous avez participé ensuite à toutes les étapes de son développement et votre ouvrage Le Climat de la Terre : quel passé pour quel avenir ? constitue un plaidoyer pour la responsabilité environnementale. Pour commencer cet entretien, pourriez-vous évoquer les étapes de votre itinéraire personnel en même temps que celles de la construction d'une science qui, avec la mise en évidence du réchauffement climatique, a soulevé une question sociétale majeure?

André Berger: J'ai commencé ma carrière en travaillant sur le problème de la pollution de l'air. À cette époque-là (1970), le climat intéressait très peu de monde. Quand j'en parlais devant mes collègues physiciens, ils rétorquaient que c'était un sujet indigne d'un physicien ou d'un mathématicien; pour eux, il s'agissait d'une question de géographie qui se résumait à faire des tables de statistiques. Pourtant, déjà dans les années 1965-1970, je faisais partie d'un groupe international de scientifiques qui tentait de construire les règles fondamentales qui régissent l'évolution du climat dans tous ses compartiments : l'atmosphère, l'océan, la glace, la lithosphère et la biosphère. Des personnalités comme Stephen Schneider ${ }^{1}$, aux États-Unis, ont joué un rôle fondamental dans cette démarche. Le climat était donc considéré, à l'origine, comme un problème de physique et de mathématiques.

Pour être plus précis, quelque temps après avoir été embauché à l'Université catholique de Louvain sur une thématique de pollution de l'air, en 1970-1971 je suis parti un an aux États-Unis pour suivre une spécialisation sur les problèmes de dynamique atmosphérique au Massachusetts Institute of Technology (MIT). J'avais besoin, pour ma thèse, d'une formation sérieuse dans cette discipline. J'avais découvert mon projet de thèse à la suite d'un congrès sur la paléoclimatologie organisé à Newcastle upon Tyne, en 1963, par un géophysicien de la Terre solide, Stanley Keith Runcorn. Je ne savais pas très bien ce qu'était la paléoclimatologie, mais la thématique $\mathrm{m}^{\prime}$ intéressait. Je venais de lire un article qui m'avait profondément choqué, dans lequel des météorologues et des géologues attaquaient les idées de Milankovitch et la théorie astronomique des climats ${ }^{2}$. En mathématicien

1 Stephen Schneider (1945-2010) : climatologue américain, professeur à l'Université de Stanford, il mit en évidence la responsabilité des gaz à effet de serre et de la pollution atmosphérique dans les changements climatiques. Membre du National Center for Atmospheric Research (NCAR) de 1972 à 1996, où il cofonda le Climate Project, il fut aussi l'un des cofondateurs du GIEC et participa, en tant que coordinateur et auteur principal, à plusieurs de ses rapports d'évaluation.

Pour plus d'informations sur Milutin Milankovitch et sa théorie astronomique des climats (ou cycles de Milankovitch), voir : http://planet-terre.ens-lyon.fr/planetterre/XML/db/ planetterre/metadata/LOM-milankovitch-2005-09-27.xml. passionné par la climatologie, je voyais là un défi extrêmement intéressant et je me suis mis en tête de construire une nouvelle solution astronomique aux variations climatiques, afin d'améliorer celles qui existaient. Je ne savais pas du tout où cela pourrait me conduire, mais je trouvais cela passionnant. C'est ainsi que j'ai commencé une thèse sur les variations du climat.

NSS : Vous n'êtes donc pas du tout astronome, au départ?

André Berger : En tant que mathématicien, on a une formation d'astronome. Mais je ne suis pas astronome au sens où les Français l'entendent. Les cours de mécanique céleste, qui font partie du cursus de mathématiques, m'ont permis d'aborder le problème de la révolution de la Terre autour du Soleil et de sa rotation autour de son axe, et ainsi d'améliorer le calcul des variations à long terme des éléments astronomiques qui sont à la base de la théorie de Milankovitch.

NSS : Pouvez-vous nous en dire plus sur Milankovitch?

André Berger : Milutin Milankovitch était d'origine serbo-croate. Il est né en 1879 à Dalj (actuellement en Croatie) et il a passé la plus grande partie de sa vie à Belgrade, où il est décédé en 1958. Au départ, c'était un ingénieur des travaux publics, métier qu'il abandonna pour se consacrer, dès le début $\mathrm{du} X X^{\mathrm{e}}$ siècle, à la recherche fondamentale sur les variations climatiques du Quaternaire. Son premier livre, publié en français par GauthierVillars en 1920, s'intitule La Théorie mathématique des phénomènes thermiques produits par la radiation solaire. Son œuvre principale, Kanon der Erdbestrahlung und seine Anwendung auf das Eiszeitenproblem, date toutefois de 1941 ; elle a été publiée en allemand à Belgrade ${ }^{3}$. Cette théorie a pour objet d'expliquer les cycles glaciaireinterglaciaire à partir des variations de l'axe de la Terre et de son orbite, variations qui sont responsables des modifications de l'énergie solaire disponible à la surface du globe. Pour ce faire, Milankovitch a introduit la notion de "saisons caloriques». Ces saisons de six mois furent malheureusement à l'origine de critiques acerbes de la part de certains météorologues. Les variations d'énergie disponibles pendant de telles saisons étant extrêmement faibles, le système climatique - prétendaient-ils - était beaucoup trop résistant pour réagir à ce genre de variations.

C'est pourquoi, dans les années 1960, après avoir recalculé avec grande précision l'énergie solaire arrivant à la surface de la Terre, j'ai introduit le calcul de l'énergie journalière, ce que Milankovitch n'avait pas fait. Cela

3 Ellea par la suite été traduite en anglais : Canon of Insolation and the Ice-Age Problem, Jerusalem, Israel Program for Scientific Translation, 1969 ; Belgrade, Texbook Publishing Compagny, 1998. 
s'imposait, car les modèles climatiques qui commençaient à être développés nécessitaient le calcul des flux d'énergie de façon continue. Il devait s'avérer, en outre, que les variations de ces insolations journalières étaient beaucoup plus conséquentes qu'on ne le pensait. Ainsi, pendant le dernier interglaciaire, la variation d'énergie au moment du solstice d'été a atteint, dans nos latitudes tempérées, quelque $25 \%$ en 10000 ans, ce qui est considérable. Passionné par la modélisation climatique, je suis alors parti au MIT. Si je voulais m'en sortir, il fallait, en effet, que je possède une formation sérieuse en dynamique atmosphérique, car à cette époque le climat se rapportait essentiellement à l'atmosphère. C'est plus tard seulement qu'on s'intéressera aux autres parties du système climatique : 1'océan, la glace, la couverture des sols et la biosphère.

NSS : Cette histoire correspond assez à la tonalité de Natures Sciences et Sociétés... Finalement, l'innovation scientifique est venue, d'abord, d'un ingénieur du génie civil et vous avez pris la suite en tant que mathématicien. Cela veut-il dire que les organisations scientifiques des disciplines sont trop rigides pour permettre l'innovation et qu'il faut qu'elle vienne de l'extérieur, avec tous les combats qu'on imagine, ou bien est-ce purement fortuit?

André Berger: En réalité, ce n'est ni l'un ni l'autre, car, à cette époque, le climat n'était pas encore une science. Resituons le contexte historique dans lequel Milankovitch a pu entreprendre ses travaux. Dans son livre Révolutions de la mer : déluges périodiques, qui date de 1842, le mathématicien français Joseph-Alphonse Adhémar explique que l'énergie qui nous vient du Soleil a varié suffisamment au cours du temps pour engendrer les grandes glaciations qui venaient d'être découvertes par le Suisse Louis Agassiz. Selon Adhémar, l'origine des glaciations devait résider dans l'existence d'hivers froids, et il a ainsi introduit les variations des trois paramètres astronomiques à la base de toute théorie expliquant les cycles glaciaire-interglaciaire, à savoir l'excentricité de l'orbite de la Terre, l'inclinaison de son axe de rotation et la précession climatique, qui reflète la variation de la distance Terre-Soleil pour une saison donnée et donc l'énergie reçue pendant chaque saison. Mais, fait incroyable pour l'époque, Adhémar a aussi esquissé la théorie du tapis roulant océanique qui redistribue l'énergie à la surface de la planète, théorie qui est aujourd'hui fondamentale pour la compréhension du climat et de ses variations passées et futures. Milankovitch connaissait les travaux d'Adhémar. Mais il a aussi été influencé par ceux d'un astronome du XIX ${ }^{\mathrm{e}}$ siècle, John Herschel, qui avait abordé le calcul de l'insolation du point de vue théorique. J'ai repris de tels calculs de l'énergie qui nous vient du Soleil à partir des intégrales elliptiques introduites par Levi W. Meech dès 1855 et par Christian Wiener en 1876. Ce travail a été publié en 1975 dans les Annales de la Société scientifique de Bruxelles. Il y a peu, j'ai repris tous ces calculs dans un article publié dans la revue internationale Quaternary Science Reviews ${ }^{4}$, qui donne accès (via un site Internet) à un programme informatique permettant le calcul précis et immédiat de toute forme d'énergie solaire reçue sur Terre.

NSS : Mais, lorsque vous vous êtes lancé dans ce travail de réhabilitation de Milankovitch, vous étiez en porte-à-faux avec toutes les convictions des géologues de l'époque concernant le Quaternaire.

André Berger : Effectivement, la nouvelle version de la théorie astronomique remettait en question un certain nombre de connaissances de l'époque, car les géologues croyaient encore à l'existence de seulement quatre glaciations (Würm, Riss, Mindel et Günz) au cours du dernier million d'années. Ce sont ces quatre glaciations que Milankovitch tentait d'expliquer, avec l'aide de quelques autres grands scientifiques dont le climatologue Wladimir Köppen et le météorologue Alfred Wegener, qui n'était autre que le gendre du premier. C'est d'ailleurs par l'intermédiaire de Köppen que Wegener et Milankovitch se sont rencontrés. Ils ont rédigé ensemble des chapitres entiers des livres de la série des Handbuch der Geophysik que Wegener a publiés avant de mourir. Ni l'un ni l'autre n'ont connu le succès de leur œuvre, alors que nul ne doute aujourd'hui de l'impact considérable qu'ils ont eu, l'un (Wegener) sur la théorie de la tectonique des plaques, l'autre (Milankovitch) sur celle de l'évolution du climat. À la fin de sa vie, Milankovitch, qui à l'origine était un bon vivant, était extrêmement déprimé de n'avoir jamais été reconnu.

NSS : Mais, pour revenir à l'idée que vous avez exprimée sur les conditions favorables à l'innovation, lorsque vous avez débuté, la climatologie n'était pas encore réellement constituée comme science?

André Berger: La climatologie a commencé d'être une science reconnue dans les années 1960-1970, fondée sur la physique et les mathématiques. Il a d'abord fallu définir ce qu'était le climat et les différentes branches complexes qui le constituent. À partir de là, les équations qui gouvernent la dynamique des différentes parties du système climatique ont pu être écrites. Parmi les disciplines qui devaient être abordées, la météorologie fut la première, car son origine remonte au début du $X^{\mathrm{e}}$ siècle. L'océanographie, avec les mesures faites dans tous les océans, la glaciologie et les explorations polaires, la biogéochimie et l'étude du cycle du carbone allaient progressivement voir le jour. Ensuite, on s'est intéressé non seulement au contenu de chacune des parties du système climatique, mais aussi aux interactions entre elles : Comment l'océan interfère-t-il avec l'atmosphère et comment l'atmosphère exerce-t-elle une influence sur l'océan?

\footnotetext{
4 Berger, A., Loutre, M.-F., Yin, Q., 2010. Total irradiation during any time interval of the year using elliptic integrals, Quaternary Science Reviews, 29, 17-18, 1968-1982.
} 
Comment le cycle du carbone se trouve-t-il imbriqué dans le système ? Quel est le rôle des précipitations dans la formation d'une calotte de glace? Il fallait des chercheurs qui n'aient pas peur de sortir de leur discipline, et il faut reconnaitre qu'aux États-Unis les scientifiques étaient moins prisonniers de disciplines individuelles. En France, sont apparus les premiers paléoclimatologues, tels que Jean-Claude Duplessy, un des premiers géochimistes de l'océan. Ensuite, il y a eu la palynologie avec Armand Pons et la dendroclimatologie. Les premières relations statistiques entre l'épaisseur d'un cerne annuel, la température et les précipitations de certains mois de l'année furent validées sur des périodes pour lesquelles des mesures climatiques instrumentales existaient et ensuite étendues à des périodes plus anciennes pour reconstituer, par modélisation inverse, le climat de celles-ci.

La dendroclimatologie a joué un rôle fondamental dans ma carrière : je venais de soutenir ma thèse en 1973, totalement inconnu, quand un de mes collègues, André Munaut, dendrochronologue à Louvain, m'a présenté à Hal Fritts, qui était à cette époque directeur du laboratoire de dendroclimatologie à Tucson (Arizona). Celuici me demanda de l'aider à construire les équations de transfert des anneaux de croissance des arbres en fonction du climat. Grâce à lui, et à son invitation à participer à un congrès à Tucson, j'ai pu rencontrer John Murray Mitchell, Jr., qui était à la National Oceanic and Atmospheric Administration (NOAA) un des grands pionniers de la climatologie moderne. C'est lui qui m'a mis en contact avec le Lamont-Doherty Geological Observatory ${ }^{5}$, où Jim Hays, John Imbrie et, peu après, Nick Shackleton (University of Cambridge, GB) tentaient de reconstituer les cycles glaciaire-interglaciaire à partir de l'analyse des sédiments du fond des océans. Leurs courbes climatiques établies à partir de l'étude géochimique des squelettes carbonatés des foraminifères extraits de carottes marines se sont montrées en parfait accord avec les forçages astronomiques que j'avais établis. C'est ainsi que j'ai commencé une deuxième carrière : je me suis intéressé à la géologie, dont j'ai dû apprendre les concepts de base. C'est ensemble que géologues et physiciens ont pu construire les premiers programmes de paléoclimatologie. J'ai, par reconnaissance, aidé Hal Fritts, et surtout André Munaut en Belgique, à construire les fonctions de transfert entre cernes de croissance des arbres et climat. Joël Guiot, qui a été mon premier étudiant physicien en thèse de doctorat, m'a épaulé dans ce travail. Il devait par la suite être accueilli par Armand Pons dans son laboratoire de Marseille. C'est là qu'ont été lancées les premières banques européennes de données en palynologie et en dendroclimatologie.

Mon cheminement avec la théorie astronomique est une longue histoire. Une fois de plus, on y découvre que

5 Columbia University, Earth Institute (New York). l'évolution de la science n'est pas nécessairement programmée. Une grande part est due aux hasards de la vie et à la possibilité qu'on se donne de saisir l'occasion. En réalité, je n'avais aucune idée de l'issue de cette recherche et, surtout, je n'imaginais pas qu'elle pourrait devenir un jour populaire. C'était un projet purement théorique, fondamental, sur les équations de la mécanique céleste, les intégrales elliptiques et la modélisation du climat, dont on parlait à peine. Ce n'était pas une recherche d'actualité et il n'y avait pas de financement pour elle. C'est donc pour gagner ma vie que je me suis occupé de pollution de l'air, à la mode à cette époque. Mais, à la fin des années 1970, j'ai pu abandonner ces travaux pour me consacrer à l'étude des climats du passé. En France, le Commissariat à l'énergie atomique (CEA) et le Bureau de recherches géologiques et minières (BRGM) avaient besoin de mes données pour étudier la stabilité des sites de stockage des déchets nucléaires. Et c'est avec eux que j'ai obtenu un de mes premiers contrats de recherche en paléoclimatologie, au début des années 1980.

NSS : Mais il y a eu tout de même un concours de circonstances extraordinaire, puisque, au moment même où vous validiez la théorie de Milankovitch, étaient publiées les courbes climatiques des longues séquences marines à partir des variations du taux d'isotopes lourds ${ }^{18} \mathrm{O}$ de l'oxygène dans les tests carbonatés des foraminifères fossiles $\delta^{18} \mathrm{O}$ et, pour la première fois, apparaissait la cohérence entre les données de terrain et la théorie ${ }^{6}$.

André Berger : Ici encore, il faut rendre hommage à John Murray Mitchell, Jr., car c'est lui qui a poussé à la renaissance de la théorie de Milankovitch, mais aussi à la quantification du problème. Hays, Imbrie et Shackleton allaient utiliser pour la première fois des techniques d'analyse spectrale pour montrer qu'on retrouvait effectivement les cycles astronomiques de 100000 ans, de 41000 ans et de 21000 ans dans les sédiments des océans profonds. En réalité, ils allaient identifier deux cycles principaux dans la bande de la précession : un cycle de 23000 ans et un cycle de 19000 ans. Cela a déçu profondément Imbrie, car il s'attendait à trouver un seul cycle de 21000 ans. Or, au même moment - les deux articles ont été publiés à quelques mois d'intervalle ${ }^{7}-$, je montrais que la précession avait en fait comme périodes fondamentales 19000 ans et 23000 ans, et que 21000 était seulement une moyenne et non pas une composante spectrale. Ce résultat a évidemment rassuré Imbrie, qui a

\footnotetext{
6 Cf. http://planet-terre.ens-lyon.fr/planetterre/XML/db/ planetterre/metadata/LOM-series-temporelles1.xml.

7 Hays, J.D., Imbrie, J., Shackleton, N.J., 1976. Variations in the Earth's orbit: Pacemaker of the Ice Ages, Science, New Serie, 194, 4270, 1121-132 (http:/ / www.jstor.org/stable/1743620?origin=JSTOR-pdf). Berger, A., 1977. Support for the astronomical theory of climatic change, Nature, 269, 44-45 (http:/ / www.nature.com/nature/journal/v269/n5623/pdf/269044a0.pdf).
} 
alors affirmé que cette double fréquence obtenue conjointement, mais totalement indépendamment, dans les données géologiques et dans les calculs purement théoriques était la première grande démonstration de la véracité de la théorie astronomique des paléoclimats.

\section{L'organisation de la science}

NSS : Vous avez produit scientifiquement dans un domaine qui allait prendre une importance considérable : le changement climatique. Ce sujet a fait l'objet d'une organisation coopérative spécifique, le GIEC, au niveau des Nations unies. Vous avez participé à sa genèse et vous avez observé ses évolutions au cours de sa déjà longue existence. Quel regard portez-vous sur cet objet « hybride»?

André Berger : J'ai participé effectivement au lancement du GIEC (rapport $n^{\circ} 1$ ) et $j^{\prime}$ en suis heureux. Je me suis, par contre, assez vite retiré de l'activité rédactionnelle des groupes de travail (Encadré), dès le second rapport. Outre le fait que de nombreux et talentueux collègues souhaitaient $s^{\prime} y$ investir, j'ai été frappé par la difficulté de gestion d'un grand nombre de participants et par le temps qu'il faut y consacrer. L'organisation interne du travail de rédaction repose d'ailleurs de plus en plus sur le recours à de jeunes chercheurs à qui l'on confie analyses et synthèses, ce qui constitue une aide mais fait prendre quelques risques. $\mathrm{Vu}$ l'ampleur des résultats obtenus de par le monde, la rédaction des rapports devient de plus en plus ardue. La mise en valeur des résultats fondamentaux est souvent empêchée par la difficulté et la quantité des processus qui gouvernent le comportement du système climatique et qu'il faut expliquer pour rester crédible. Les Groupes II et III ont été créés à la suite de demandes plus politiques, justifiées par la nécessité de se pencher sur les impacts du réchauffement global et sur les moyens de les réduire. L'intégration finale des rapports des trois groupes reste un problème difficile.

NSS : Au même moment, quasiment, vous avez été un moteur dans l'élaboration européenne des premiers programmes-cadres de recherche, dans le domaine qui vous concerne. Depuis, l'évolution nette de ces programmes a pu souvent désorienter les chercheurs de base. Le cadre européen est-il encore, voire toujours, un endroit où une recherche de qualité et de passion peut se développer?

André Berger: J'ai participé avec enthousiasme au lancement des premiers programmes ; le petit groupe que nous constituions (Klaus Hasselmann, Jean-Claude Duplessy, Tom Wigley, Roberto Fantechi et moi-même) était un groupe de réflexion créatif qui s'intéressait d'abord à la recherche fondamentale portant sur les processus qui régissent le comportement du système. La nécessité d'aborder l'étude des impacts est venue progressivement, en même temps qu'une approche beaucoup moins directive de la question. La cassure résulte des orientations données à la suite des conflits d'intérêts associés au mandat d'Édith Cresson à la Commission européenne - elle y était à l'époque commissaire. Il s'en est suivi une transformation des appels d'offres selon une approche bottom-up; l'explosion de projets qui en a résulté, d'une part, et l'abandon de projets de base au profit d'objectifs immédiats, d'autre part, ont considérablement modifié et affaibli le mode de sélection des projets et accentué les aspects administratifs au détriment d'une science ambitieuse. Je me suis retiré complètement du dispositif à la fois comme animateur et comme coordinateur et je suis retourné exclusivement à ma recherche.

NSS : Si nous comprenons bien, il y a deux changements importants qui vont modifier le paysage scientifique: d'une part, un abandon des recherches de base pour une finalisation faussement utile ; d'autre part, un amoindrissement de la fonction d'orientation, les programmes scientifiques suivant de plus en plus les offres du marché.

André Berger: Heureusement, au début du $\mathrm{XXI}^{\mathrm{e}}$ siècle, en particulier sous l'impulsion du prix Nobel Paul Crutzen, la Commission européenne a créé le Conseil européen de la recherche (European Research Council $\left.[E R C]^{8}\right)$. Les appels d'offres pour les Advanced Grants furent lancés en 2007, privilégiant la recherche fondamentale et l'excellence scientifique. J'ai eu la chance d'avoir mon projet retenu et classé « top », le financement de ce projet couvrant la période 2008-2013. Grâce aux budgets qui m'ont été alloués, j'ai pu engager quatre docteurs ès sciences pour étudier la mousson et le climat d'il y a 500000 ans. Les lœss et les sols de cette époque, en Chine, permettent de supposer que la mousson de l'Est asiatique y fut exceptionnelle, bien que le climat au sein duquel elle se manifesta fût celui d'un interglaciaire frais. J'avais en réalité commencé cette recherche dès 2005, grâce à une collaboration avec des chercheurs chinois. Cette collaboration est toujours en cours et c'est Qiuzhen Yin, chercheure à l'Université catholique de Louvain, qui en assure le suivi au sein de mon projet ERC, où sa contribution est déterminante. Certes, les sommes consenties à l'ERC sont relativement petites par rapport aux montants globaux du budget de la recherche de l'Union européenne, mais j'y ai retrouvé un fonctionnement beaucoup plus souple, laissant libre cours à la passion, à l'originalité et à l'innovation - même si, malheureusement, le contrôle financier demeure des plus tatillon.

\footnotetext{
8 Voir l'entretien de Pieter Leroy avec Helga Nowotny, présidente actuelle : « Helga Nowotny: an itinerary between sociology of knowledge and public debate », Natures Sciences Sociétés, 17, 1 (2009), 57-64.
} 


\section{Encadré. Le Groupe d'experts intergouvernemental sur l'évolution du climat (GIEC)}

\section{Historique}

Le principe d'une influence des émissions de gaz à effet de serre sur le climat a été découvert au XIX ${ }^{\mathrm{e}}$ siècle, et confirmé au $X X^{e}$ siècle à la suite de nombreux développements techniques et scientifiques : multiplication des observations, y compris par les premiers satellites, apparition d'ordinateurs à même de réaliser des simulations du système climatique... Plusieurs programmes de recherche en matière de climat ont été organisés à l'échelle mondiale, dont notamment le Programme de recherche sur l'atmosphère globale (GARP), mis en place en 1967 par l'Organisation météorologique mondiale (OMM) en collaboration avec le Conseil international des unions scientifiques (CIUS - en anglais, ICSU).

Les débats qui suivirent ont conduit, en 1988, à la création du Groupe d'experts intergouvernemental sur l'évolution du climat (GIEC - en anglais IPCC), sous l'égide de l'OMM et du Programme des Nations unies pour l'environnement (PNUE). La mission du GIEC est « d'évaluer, sans parti pris et de façon méthodique, claire et objective, les informations d'ordre scientifique, technique et socio-économique qui nous sont nécessaires pour mieux comprendre les fondements scientifiques des risques liés au changement climatique d'origine humaine, cerner plus précisément les conséquences possibles de ce changement et envisager d'éventuelles stratégies d'adaptation et d'atténuation ${ }^{\mathrm{a}}$. » La première réunion du GIEC eu lieu du 9 au 11 novembre 1988 à Genève, sous la présidence du météorologue suédois Bert Bolin ${ }^{\mathrm{b}}$; trois groupes de travail ont été établis :

- Groupe I (présidé par sir John Houghton, chef de l'Office météorologique anglais) : évaluation des informations scientifiques disponibles sur les changements climatiques ;

- Groupe II (Dr Yuri Izrael, chef du service Hydrométéorologique, URSS) : évaluation des conséquences environnementales et socioéconomiques des changements climatiques ;

- Groupe III (Dr Frederick Bernthal, secrétaire d'État adjoint, US Department of State) : formulations de stratégies de réponse.

Au cours de cette première réunion, un programme de travail fut aussi discuté et il fut décidé que le premier rapport (First Assessment Report [FAR]) serait publié avant la deuxième Conférence mondiale sur le climat, prévue en 1990. L'Assemblée générale des Nations unies prit connaissance de ce rapport lors de sa $45^{\mathrm{e}}$ session (1990) et décida d'entamer des négociations pour qu'une Convention-cadre sur les changements climatiques puisse être préparée avant la Conférence des Nations unies sur l'environnement et le développement, prévue en juin 1992 à Rio de Janeiro. Elle y sera effectivement adoptée.

Le GIEC a produit, à ce jour, quatre rapports d'évaluation, qui représentent une synthèse de l'ensemble de la problématique des changements climatiques. Le cinquième rapport, actuellement en cours de rédaction, paraîtra en 2013-2014.

Pour son travail et ses « efforts de collecte et de diffusion des connaissances sur les changements climatiques provoqués par l'homme, et pour poser les fondements des mesures nécessaires pour lutter contre ces changements ${ }^{\mathrm{c}}$ ", le GIEC a reçu, conjointement avec $\mathrm{Al}$ Gore, le prix Nobel de la paix en 2007.

\section{Domaines d'expertise : la place des sciences sociales}

Le GIEC prend en compte toutes les études faisant preuve de rigueur scientifique. Son évaluation des connaissances est basée sur un maximum de disciplines et avis pertinents et se fait avec la plus grande objectivité possible.

Les rapports d'évaluation sont divisés en trois parties, correspondant aux trois Groupes de travail, dont les domaines d'expertise ont par ailleurs évolué depuis leur création : le premier volume porte sur le fonctionnement physique du système climatique et les variations du climat passées et à venir ; le second, sur l'étude de la vulnérabilité face aux risques liés aux changements climatiques et sur les stratégies possibles d'adaptation ; le troisième s'intéresse aux stratégies de réduction des émissions de gaz à effet de serre. Les sciences économiques et sociales sont donc particulièrement importantes pour les travaux des Groupes II et III.

Le Groupe II s'occupe des questions concernant la vulnérabilité des systèmes socioéconomiques et naturels aux changements climatiques, les conséquences négatives et positives de ces changements et les possibilités de s'y adapter. Tout comme la capacité d'adaptation, la vulnérabilitéd des personnes dépend en partie du contexte socioéconomique dans lequel elles vivent, notamment de facteurs tels que l'accès aux richesses, la présence d'infrastructures, les échanges économiques, les modes d'alimentation...

Le Groupe III évalue les solutions envisageables pour limiter les émissions de gaz à effet de serre ou atténuer de toute autre manière les changements climatiques. Il étudie pour cela les liens entre activité économique et émissions, les coûts associés aux réductions d'émissions, les instruments économiques à la disposition des décideurs, etc. 
Les Groupes II et III traitent donc de questions spécifiques pour lesquelles une connaissance du développement économique et social est indispensable ; mais ils s'intéressent aussi à des aspects de plus en plus importants pour le développement lui-même. Pour tenter de répondre à ces questions, le GIEC utilise des " scénarios » décrivant différents futurs possibles, sur la base de la littérature scientifique, en matière démographique, technologique, comportementale, économique... Une part du travail de synthèse consiste alors à relier ces scénarios socioéconomiques aux émissions qui ont une action sur le climat.

Le rôle du GIEC est d'informer les décideurs sur les différents futurs possibles, mais sans faire de choix politiques. Chaque rapport fait ainsi l'objet d'un résumé à leur intention. Il s'agit de rendre compte, dans un langage clair et accessible, des résultats des expertises scientifiques. Là encore, il est fait largement appel aux sciences sociales.

André Berger et Jean-Pascal van Ypersele

a Cf. http://www.ipcc.ch/home_languages_main_french.shtml\#.UHbX7UJrgfk.

Report of the first session of the WMO/UNEP Intergovernmental Panel on Climate Change: http://www.ipcc.ch/meetings/ session01/ first-final-report.pdf.

c Cf. http://www.un.org/french/aboutun/nobelprize/2007.shtml.

d Cf. le Rapport spécial sur la gestion des risques de catastrophes et de phénomènes extrêmes pour les besoins de l'adaptation au changement climatique (SREX), réalisé par les Groupes I et II.

\section{Interdisciplinarité entre sciences de la nature et sciences sociales}

NSS : NSS a mis au centre de ses réflexions théoriques et pratiques l'interdisciplinarité entre sciences de la nature et sciences de la société. Dans ce domaine, le changement climatique, par son impact sur les sociétés humaines, paraît intéressant : en témoigne notamment l'existence des Groupes II et III du GIEC. Que pouvezvous nous dire de votre vécu de cette situation et de votre engagement?

André Berger: L'étude du changement climatique requiert un travail multidisciplinaire qui mobilise des savoirs élaborés et complexes ; il est dès lors important que l'on puisse se parler et se comprendre, même si on n'appartient pas à la même discipline. Ma recherche personnelle sur les climats anciens n'inclut pas les impacts du changement climatique sur l'environnement et la société. Pour autant que je puisse en juger, les Groupes II et III du GIEC tentent d'utiliser au mieux les résultats $d u$ Groupe I, chargé de comprendre l'évolution du climat, mais une collaboration intégrée reste difficile, les sciences en jeu (naturelles, sociales et humaines) étant basées sur des concepts différents et utilisant des méthodes pas toujours comparables.

NSS : Certes, mais comment nourrir des modèles d'impacts dont les résultats pourraient être ensuite le support d'actions et de décisions?

André Berger: $\mathrm{Si}$, pour des processus naturels (cycle de l'eau, écosystèmes...), on peut construire des modèles basés sur des lois de la physique, de la chimie, de la biologie, de l'écologie, etc., ce n'est pas le cas dans le domaine des sciences sociales. Le couplage entre climatologie et sciences économiques et sociales ne peut que déboucher sur un accroissement important des incertitudes. L'exemple de l'effort intégré du MIT (en limitant la participation des sciences sociales à l'économie) est intéressant de ce point de vue. Il met en évidence le temps nécessaire pour que les disciplines se comprennent et puissent ensuite travailler ensemble. Quant à moi, je préfère me consacrer à la recherche fondamentale dans une discipline que je pense bien maîtriser. J'espère ainsi pouvoir cerner les mécanismes climatiques moins bien connus et en améliorer la représentation de façon à limiter les incertitudes, au moins pour la modélisation du climat et de ses variations.

NSS : Cela dit, l'opportunité d'adaptations repose sur une comparaison entre les coûts de celles-ci et les dommages subis ; c'est, semble-t-il, dans ce sens-là qu'évolue le travail du Groupe II.

André Berger : C'est évidemment un choix personnel. Mon collègue de Louvain-la-Neuve, Jean-Pascal van Ypersele, qui a présidé le Groupe II, est plus optimiste quant à l'apport des sciences humaines dans le problème du réchauffement global (Encadré). L'élaboration des scénarios de consommation énergétique future nécessite, bien sûr, une collaboration intense entre sociologues, démographes, politiques, ingénieurs, géologues et énergéticiens pour estimer les disponibilités et les besoins futurs en quantité d'énergie, en sources et en moyens d'approvisionnement.

NSS : Si le partenariat est encore loin, il faut le préparer, et vous avez insisté sur la nécessité de prérequis pour aller vers l'autre. Quelle vision avez-vous de la présentation de la question climatique dans les formations d'étudiants que vous connaissez ?

André Berger: Il est évident qu'on ne peut être spécialiste en tout. La formation d'un chercheur en 
climatologie est si exigeante au niveau de la discipline elle-même qu'on peut difficilement imaginer la doubler d'autres formations dans des disciplines totalement différentes. La collaboration est donc inévitable. Elle ne sera toutefois efficace que si un langage commun existe et si les incertitudes sont bien ciblées et quantifiées.

\section{La controverse avec les climatosceptiques et l'Académie des sciences}

NSS : Comme vous le savez, les conclusions du GIEC ont été contestées et cette contestation a pris une résonnance particulière en France avec ce qu'on peut appeler «l'affaire Allègre ». Il y a une façon de prendre le problème au départ, qui est de se demander : Quelles peuvent avoir été les faiblesses de l'organisation des climatologues français pour donner le flanc à ces attaques? N'ont-ils pas été étroitement corporatistes?

André Berger : Il ne faut pas retourner le problème. Ce n'est pas parce que le GIEC est attaqué par quelques personnes qui n'ont d'autre but que de faire parler d'elles qu'il faut l'accuser, pas plus que les climatologues français. J'ai passé une grande partie de ma vie à collaborer avec les chercheurs français et je ne vois pas en quoi on pourrait les accuser de corporatisme.

NSS : Formulons la question autrement et prenons un exemple significatif de ces affrontements. Dans une émission de télévision, qui avait une écoute assez importante, Claude Allègre s'est trouvé confronté à un représentant de la science, en l'occurrence Valérie Delmotte, et à trois ou quatre citoyens qui avaient eu à souffrir d'une tempête ou d'une inondation. On percevait l'honnêteté de V. Delmotte, qui restait dans le champ de sa science, et la roublardise de $C$. Allègre, capable de changer de registre très rapidement entre ses prétentions scientifiques et une sorte d'interdisciplinarité personnelle, sautant des évolutions climatiques à des problèmes de taxes, ce qui noyait le débat.

André Berger : Oui, mais tout le monde n'est pas au courant des motifs d'Allègre. Précisons d'abord qu'il n'est pas climatologue. C'est un spécialiste de la Terre solide et en particulier des chambres magmatiques. On pourrait se souvenir de ses querelles antérieures avec le vulcanologue Haroun Tazieff. Il est certain que tous, nous préférons entendre que tout va pour le mieux dans le meilleur des mondes plutôt que l'inverse. C'est précisément le discours que tiennent certains climatosceptiques, tels que Claude Allègre en France, Bjorn Lomborg au Danemark (même s'il a changé son fusil d'épaule et demande à présent que la lutte contre le changement climatique soit une priorité pour les gouvernements) ou encore Fred Singer aux États-Unis. Il est clair que le citoyen préfère entendre ce discours rassurant plutôt que celui sur le « danger » proféré par le GIEC.
NSS : Oui, mais alors on a d'un côté les lanceurs d'alerte, et vous en faites partie, et de l'autre côté les gens qui utilisent le doute pour que, finalement, tout reste pareil.

André Berger : Allègre ne sème pas le doute, il affirme bien souvent des hérésies (pas toujours) pour qu'on l'écoute. C'est une sérieuse différence. Sa finalité n'est pas d'essayer de faire comprendre un problème difficile, elle est d'attirer l'attention sur lui. Qu'Allègre et Singer soient sceptiques ne me dérange pas. Ce qui me dérange profondément, c'est qu'on leur donne autant la parole, voire plus, qu'à la communauté de ceux qui en savent beaucoup plus et travaillent dur pour comprendre et, prudemment, transférer l'information à la société. Pourquoi leur demande-ton leur avis? En cas de confrontation avec un spécialiste, comme ces climatosceptiques ont le verbe haut, ils l'emportent généralement. Au mieux, la conclusion de fin d'émission sera que les scientifiques ne sont pas d'accord entre eux (et donc pourquoi devrais-je les croire ?). Le spectateur, le citoyen, met alors sur un pied d'égalité la pensée isolée des climatosceptiques et celle des chercheurs spécialistes du climat représentés, dans le cas que nous évoquions, par Valérie Delmotte. Au pire, le spectateur sera convaincu qu'Allègre a raison, car c'est quand même une grande personnalité : ancien ministre, professeur d'université, membre de l'Académie des sciences... Il nous dit que tout va bien, donc tout va bien. C'est inévitable. Les médias sont en partie responsables, car, pour leur audimat, ils donnent la parole à ces contradicteurs. Le show l'emporte sur une recherche de vérité et une tentative d'expliquer un problème qui reste délicat et difficile.

NSS : Même si cela n'a pas été très médiatisé, la controverse a atteint l'Académie des sciences, dont Claude Allègre est membre et vous-même, membre associé étranger. Quel est le rôle d'une organisation de ce type-là dans ce genre de débats ? Comment évaluez-vous son implication dans ce cas particulier?

André Berger : Une des fonctions de l'Académie des sciences, qu'elle reconnaît elle-même, est précisément de valoriser auprès du grand public l'information scientifique. Comme le Collège de France. Ce sont les deux grandes institutions françaises qui assument la responsabilité de faire comprendre à la société l'importance de certains problèmes. L'Académie des sciences se devait donc d'aborder la question du changement climatique. Mais, dans le cas qui nous concerne, il est clair qu'elle a manqué son objectif à deux reprises. Quand Allègre a attaqué les climatologues de façon véhémente et non fondée, elle aurait pu réagir plus fermement (cela aurait aussi pu être le cas du CNRS). Ensuite, la première réunion (en octobre 2009), où on m'avait proposé d'être rapporteur, aurait pu être mieux organisée. J'avais accepté cette responsabilité avec enthousiasme, parce que je croyais en une réunion-débat où les scientifiques qui 
connaissent le problème viendraient le présenter, en faire une synthèse et répondre aux questions des journalistes. Malheureusement, une semaine avant la réunion, j'ai appris qu'il était question de donner la parole aux climatosceptiques. Je ne pouvais accepter une telle décision, car je connais parfaitement leurs argumentations, pour moi sans valeur; et, surtout, je préconisais une issue contraire à l'objectif initial, qui était de faire le point de nos connaissances et incertitudes sur le réchauffement global et son lien avec les activités humaines. Fort heureusement, mes collègues français ont relevé le défi et sauvé ce qui pouvait l'être.

Enfin, il y a eu une nouvelle réunion, en octobre 2010, à laquelle j'ai cette fois accepté de participer. Comme membre associé étranger, je dois avouer avoir peu participé aux réunions de l'Académie des sciences. Étant de ce fait peu connu de la plupart des membres, j'ai pu observer la réunion presque dans l'anonymat. Disons d'emblée que le rapport qui en est sorti est excellent et largement meilleur que ce que laissaient présager les débats euxmêmes. Première observation : deux des spécialistes sont intervenus de manière suffisamment agressive, utilisant la même passion et le même vocabulaire que ceux qui les « attaquaient »-ils ont étéà la hauteur. J'ai jugéles autres interventions trop molles. J'en ai été étonné et je pense que les scientifiques devraient vaincre leur timidité et apprendre à débattre. Ma seconde observation porte sur l'organisation des débats: apparemment, le compte rendu des débats a été confié à des non-spécialistes du climat, ce qui m'a singulièrement frappé. Il n'est guère facile, en effet, quelle que soit sa propre valeur, de synthétiser une information souvent spécialisée et parfois nuancée quand on ne maîtrise pas parfaitement la discipline. Commentaires privés entre les participants, questions parfois naïves et réponses manquant de virulence m'ont laissé penser que le rapport allait être catastrophique. Ce ne fut pas le cas, et je m'en suis réjoui, m'étant laissé dire qu'une très bonne collaboration avec les climatologues lors de la rédaction avait finalement conduit au rapport que l'on connaît.

NSS : L'Académie des sciences a aussi donné un signal fort en cooptant Édouard Bard.

André Berger: Ce fut une excellente nouvelle d'apprendre cette cooptation d'Édouard Bard, par ailleurs totalement méritée. C'est un signal fort de l'Académie des sciences, qui disqualifie les climatosceptiques et redonne au climat ses lettres de noblesse.

NSS : Richard Lindzen est-il un climatosceptique américain?

André Berger : Lindzen est effectivement un climatosceptique américain. Il n'est pas à comparer avec Allègre, Coutillot ou Singer, parce que c'est un météorologue de qualité. Professeur de météorologie dynamique au MIT, il connaît très bien le problème du climat et ses remarques poussent parfois, sinon souvent, à une réflexion profonde. Je pense que Lindzen a été instrumenté par le monde politique américain, qui ne désirait nullement voir le réchauffement du climat prendre le pas sur l'économie et l'indépendance de décision du peuple américain.

\section{Mes opinions et ma communication}

NSS : Scientifique ardent défenseur de la recherche fondamentale, vous avez néanmoins constamment communiqué à partir de votre cœur de compétences dans des réunions diverses ou sur des supports variés : ce n'est pas très fréquent ! Vous vous êtes immergé dans la jungle des médias au cours de débats souvent difficiles. Que retirezvous de cette action permanente, et sûrement usante?

André Berger : Je suis résolument optimiste quant aux capacités de la science et des technologies à relever le défi du réchauffement global et de ses conséquences sur l'environnement. La science nous permettra de comprendre et la technologie, de trouver des solutions. Par contre, je suis moins optimiste en ce qui concerne le comportement de la société. Les exemples ne manquent pas de l'impossibilité d'infléchir l'accroissement des émissions de gaz à effet de serre et autres destructions de l'environnement. Utilisation accrue des véhicules individuels de transport, déboisement intensif de la forêt tropicale, pollution en tout genre, manque de discernement entre vérité et mensonge lié à une culture scientifique insuffisante ne portent pas à l'optimisme et ne sont guère prometteurs d'avenir. Au cours des trente dernières années, je me suis fortement investi dans la communication auprès du grand public. J'ai pu me rendre compte, d'une part, de la sagesse des gens et de leur réel désir de connaître, mais, d'autre part, de leur peu de persévérance dans une tentative de changer leurs habitudes, énergétiques en particulier, et de la difficulté qu'ils ont à faire la distinction entre ce qui est fondamental et ce qui l'est moins, voire dérisoire. Ils sont beaucoup plus attentifs à la publicité et à leur confort personnel qu'aux recommandations de la science.

NSS : On est frappé par votre perception de cette quasi-certitude d'aller dans le mur et de l'incapacité des sciences sociales et humaines à prendre en charge réellement cette question : cela explique-t-il votre désintérêt pour une science finalisée et l'interdisciplinarité qu'elle implique?

André Berger : Ce constat m'est imposé, il n'est pas délibéré. Je ne demanderais pas mieux que de pouvoir faire confiance aux sciences sociales et humaines. Malheureusement, peu d'exemples pratiques me donnent cette confiance. Je ne suis pas spécialiste de ce domaine et ne désire donc porter aucun jugement de valeur. J'attends, et j'espère, simplement que le dialogue 
s'intensifie entre sciences naturelles et sciences sociales et humaines. Dans cette perspective, l'éducation sous toutes ses formes joue un rôle capital. Ainsi, la prise de conscience individuelle, que j'évoquais précédemment, ne peut s'opérer sans un enseignement adapté à tous les âges. Il s'agirait d'un enseignement conduisant à une éthique de l'environnement, dont l'Université devrait être un moteur. Le problème des interactions humaines avec la géosphère et la biosphère impose, $d^{\prime}$ 'une part, la remise en question des programmes d'enseignement et de l'organisation de la recherche - cela concerne principalement la collaboration entre disciplines - et, d'autre part, la formation à long terme des chercheurs. La reconnaissance de la pluridisciplinarité de la recherche pour la santé de la planète est une nécessité urgente, étant donné la structure du système climatique et les multiples problèmes que soulève la pollution globale. La formation à long terme devrait assurer une préparation efficace aux chercheurs appelés à résoudre les problèmes que nous commençons à percevoir, mais dont nous avons la certitude qu'ils s'amplifieront au cours du temps. Il faut espérer que l'Université décide d'accepter ce rôle et d'investir dans ce qui représente un défi majeur face à la plus grande expérience géophysique involontaire jamais déclenchée par l'homme. L'avenir du monde dépend des décisions que nous allons prendre, de l'organisation des peuples, de notre sens de la responsabilité et, enfin, du savoir et du civisme de chacun. Le défi à relever est énorme. Il nous faut travailler ferme, main dans la main, jour après jour, si nous voulons faire face. 